\title{
Trial Inclusion Exclusion Criteria Rule
}

National Cancer Institute

\section{Source}

National Cancer Institute. Trial Inclusion Exclusion Criteria Rule. NCI Thesaurus. Code C83443.

An axiom that indicates the inclusion and exclusion criteria for possible study subjects. 